\title{
DISTANCIAMENTO SOCIAL E REPERCUSSÕES OCUPACIONAIS DA PANDEMIA PARA CUIDADORAS DE CRIANÇAS E ADOLESCENTES
}

DOI: 10.22289/2446-922X.V7N2A19

\author{
Marina Batella Martins ${ }^{1}$ \\ Bruna Lídia Taño \\ Marina Vilaça Cavallari Machado
}

\section{RESUMO}

O presente estudo objetivou identificar o impacto do distanciamento social provocado pela pandemia da COVID-19 em relação ao perfil ocupacional de cuidadoras de crianças e adolescentes acompanhados por uma equipe multiprofissional. 34 cuidadoras participaram por meio de questionário composto por 31 questões. Os dados quantitativos foram analisados descritivamente e foi realizado análise de conteúdo para as perguntas de grade aberta. Os resultados desse estudo apontam para a intensificação do papel de cuidadora, ausência da participação em atividades de lazer e a importância do envolvimento em atividades significativas que apoiam e sustentam a saúde. As participantes indicaram estratégias que foram efetivas para o enfrentamento dos impactos provocados pela pandemia, como atividades de lazer e prevenção ao coronavírus. Além disso, observa-se que tais estratégias foram desenvolvidas, em sua maioria, pelas próprias participantes, em redes sociais ou internet.

Palavras-chave: Terapia Ocupacional; COVID-19; Desenvolvimento Infantil.

\section{SOCIAL DISTANCING AND OCCUPATIONAL REPERCUSSIONS OF THE COVID-19 PANDEMIC FOR CAREGIVERS OF CHILDREN AND ADOLESCENTS}

\section{ABSTRACT}

This study aimed to identify the impact of social distancing caused by the COVID-19 pandemic on the occupational profile of children and adolescents caregivers accompained by a multidisciplinary team. 34 caregivers participated by fulfilling a questionnaire composed of 31 questions. Quantitative data were analyzed descriptively and the content analysis was performed by open grid questions. The results of this study point to the intensification of the caregiver's role, absence of participation in leisure activities and the importance of involvement in significant activities that support and sustain health. Participants indicated strategies that were effective in coping with the impacts caused by the pandemic, such as leisure and prevetion activities. Further,

\footnotetext{
${ }^{1}$ Endereço eletrônico de contato: marinabatella@gmail.com

Recebido em 09/08/2021. Aprovado pelo conselho editorial para publicação em 22/10/2021.
} 
it was observed that theses strategies were developed by the participants themselves, on the social networks or internet.

Keywords: Occupational Therapy; COVID-19; Child Development.

\section{DISTANCIAMIENTO SOCIAL Y REPERCUSIONES OCUPACIONALES DE LA PANDEMIA PARA LAS CUIDADORAS DE NIÑOS Y ADOLESCENTES}

\section{RESUMEN}

Este estudio investigó los efectos de la pandemia de COVID-19 en la vida de las cuidadoras de niños y adolescentes con necesidades específicas de salud, identificando el impacto del distanciamiento social en el perfil ocupacional de las cuidadoras de niños y adolescentes acompañados por un equipo multiprofesional. En total participaron 34 cuidadoras mediante un cuestionario compuesto por 31 preguntas. Los datos cuantitativos se analizaron descriptivamente y se realizó un análisis de contenido para preguntas de cuadrícula abierta. Los resultados de este estudio apuntan a la intensificación del rol de cuidador, la ausencia de participación en actividades de ocio y la importancia de involucrarse en actividades significativas que apoyan y mantienen la salud. Los participantes indicaron estrategias que fueron efectivas para hacer frente a los impactos causados por la pandemia, como actividades de ocio y prevención del coronavirus. Además, se observa que dichas estrategias fueron desarrolladas, en su mayor parte, por los propios participantes, en las redes sociales o en internet.

Palabras clave: Terapia Ocupacional; COVID-19; Desarrollo Infantil.

\section{INTRODUÇÃO}

A emancipação e autonomia de pessoas que apresentam alterações físicas, sensoriais, psicológicas, mentais e/ou sociais são foco da terapia ocupacional, profissão da área da saúde, da educação e do campo social (Barros, Lopes, \& Galheigo 2020). No domínio da profissão, saúde e bem-estar são considerados influenciados pela escolha, controle e capacidade de se envolver nas ocupações da vida cotidiana (Kristensen \& Petersen, 2015).

Considerando a centralidade da ocupação como objeto da terapia ocupacional, cabe aprofundar seu entendimento, tendo em vista que se trata de um termo multifacetado e polissêmico, que possui diferentes significados de acordo com contextos sociais, históricos e culturais (Costa, Oliveira, Corrêa, \& Folha, 2017).

De acordo com o documento "Estrutura da Prática da Terapia Ocupacional: domínio \& processo", as ocupações são atividades cotidianas que as pessoas realizam para ocupar o tempo, trazer significado e propósito para a vida, incluindo as atividades que as pessoas precisam, querem e se espera que façam. Elas são fundamentais para a saúde, identidade e Rev. Psicol Saúde e Debate. Out., 2021:7(2): 295-313. 
senso de competência, e compreendem as atividades de vida diária (AVDs), atividades instrumentais de vida diária (AIVDs), gestão da saúde, descanso e sono, educação, trabalho, brincar, lazer e participação social (AOTA, 2020).

As ocupações desempenhadas são frequentemente associadas aos papéis ocupacionais, definidos historicamente como um conjunto de comportamentos moldados pela cultura e pelo contexto que influenciam a identidade ocupacional. $O$ desempenho das ocupações humanas acontece dentro de rotinas, que podem ser compreendidas como uma sequência estabelecida de ocupações que estruturam a vida diária, podendo contribuir para a promoção da saúde (AOTA, 2020).

Para Costa, Oliveira, Corrêa e Costa (2017) as ocupações são compreendidas na perspectiva da ciência ocupacional como o fazer diário em que as pessoas são envolvidas e que tem as dimensões, forma, função e significados definidos e observados em um contexto cultural. Sendo assim, cada indivíduo irá experienciar suas ocupações de uma forma, mesmo compartilhando um determinado contexto sócio-histórico.

Tais significados podem ser compreendidos através do perfil ocupacional, que se caracteriza por um conjunto de informações relacionadas à história de vida do sujeito e de seu grupo. Tais dados são obtidos através de um processo colaborativo entre profissional e o indivíduo, e expressam a experiência subjetiva da vida da pessoa, interesses, valores e necessidades (AOTA, 2020). Assim, o levantamento do perfil ocupacional colabora para o entendimento do contexto vivenciado pela pessoa e na identificação dos desejos e prioridades de acordo com o que é significativo para o sujeito e/ou coletivo.

Com base no exposto, percebe-se que os fatores sociais, históricos, econômicos e culturais influenciam a forma como as pessoas desempenham suas ocupações, logo cada sujeito irá vivenciar um determinado período no espaço-tempo de forma singular e subjetiva, de acordo com as condições sociais, históricas, políticas, econômicas, culturais, comunitárias e pessoais que se apresentam naquele momento (Salles \& Matsukura, 2016). Assim acontece com o surgimento da pandemia da COVID-19.

Trata-se de uma doença que provoca um quadro clínico que varia de casos assintomáticos até manifestações respiratórias graves, sendo os principais sintomas: tosse, febre, dor de garganta e dificuldade para respirar (Brasil, 2020). A transmissão ocorre pela exposição a gotículas contaminadas, através do contato entre pessoas ou com superfícies, sendo o distanciamento social a principal medida de prevenção (OPAS, 2020).

No contexto da pandemia da COVID-19, as medidas de prevenção à doença, em especial o distanciamento social, trouxeram consequências para o cotidiano da população, que 
repercutem de formas diversas na vida dos indivíduos, de acordo com gênero, classe social, papéis ocupacionais e outros fatores.

Estas medidas implicaram no fechamento de diversos locais da comunidade, como escolas e comércios, e alteraram a dinâmica de trabalho das pessoas, devido à necessidade de adoção de novas regras no ambiente de trabalho presencial e ao crescimento da modalidade de trabalho remoto, que ocasionou a piora no acesso aos serviços públicos como transporte, saúde e educação, bem como na maior precarização das relações de trabalho. Verificou-se então a partir deste contexto o agravamento das desigualdades sociais e o aumento do número de famílias em condições de vulnerabilidade social (Fiocruz, 2020).

Estudo realizado no Estado do Espírito Santo identificou maior letalidade da COVID-19 nas pessoas com deficiência, reforçando a necessidade de acesso às informações sobre a prevenção da doença por essa parte da população (Maciel et al., 2020). O estudo apontou maior vulnerabilidade das pessoas com deficiência quando comparadas à população geral, considerando que elas podem apresentar maior dificuldade para acessar as informações de prevenção, transmissão e tratamento e para aderir aos hábitos preventivos (Maciel et al., 2020).

No contexto do cuidado domiciliar, é comum que a atribuição de cuidador principal seja assumida por um integrante da família, sendo a maioria do gênero feminino. Assim, com o advento da pandemia, é possível observar um aumento das tarefas atribuídas à mulher, que desempenha diversas atividades relacionadas aos seus papéis ocupacionais. Ao considerar as demais atividades desempenhadas diariamente pelas mulheres que cuidam em tempo integral, é preciso observar o risco de aumento da sobrecarga das cuidadoras, que impacta diretamente na qualidade de vida. Tal situação é intensificada ao considerarmos as cuidadoras de crianças/adolescentes com demandas específicas de saúde (Ballarin, Benedito, Krön, \& Christovam, 2016; Araújo, Dutra, Freitas, Guedes, Souza, \& Baptista, 2019).

Com base no exposto, faz-se necessário investigar os efeitos da pandemia da COVID-19 na vida de cuidadores de crianças e adolescentes com necessidades específicas de saúde, a fim de compreender o impacto das medidas de prevenção e desenvolver estratégias de enfrentamento. Assim, o presente estudo objetivou identificar o impacto do distanciamento social provocado pela pandemia da COVID-19 em relação ao perfil ocupacional de cuidadoras de crianças e adolescentes acompanhados por uma equipe multiprofissional.

\section{MATERIAIS E MÉTODOS}

Trata-se de um estudo descritivo, de abordagem quanti-qualitativa, de análise do impacto do distanciamento social, ocasionado pela COVID-19, no perfil ocupacional de cuidadorasi de Rev. Psicol Saúde e Debate. Out., 2021:7(2): 295-313. 
crianças e adolescentes com necessidades específicas de saúde atendidos pela equipe da Residência Multiprofissional no Ambulatório de Pediatria de um hospital público de uma capital do Brasil.

O Ambulatório de Pediatria atende crianças e adolescentes que apresentam diversas condições de saúde como doenças crônicas, atraso no desenvolvimento neuropsicomotor, e síndromes, e possui o projeto Follow up (seguimento para acompanhamento aos recém-nascidos prematuros). Esse público é acompanhado pelo Programa de Residência Multiprofissional na Saúde da Criança e do Adolescente, com frequência que varia entre semanal a mensal. $O$ público-alvo atendido pela residência multiprofissional é encaminhado pelos profissionais das clínicas do ambulatório. Com a pandemia da COVID-19, os atendimentos foram suspensos entre março e julho de 2020, e a partir de agosto a equipe da residência multiprofissional retornou de maneira gradual. Os atendimentos foram retomados em menor número, com intervalos maiores, adotando as medidas de prevenção recomendadas. Para tanto, foi priorizado o atendimento aos casos de crianças e adolescentes que já estavam se deslocando ao ambulatório para consultas médicas. Portanto, é possível hipotetizar que houve uma ruptura brusca no cotidiano dos cuidadores de crianças e adolescentes acompanhados neste setor, tanto pela interrupção da assistência prestada, quanto pela necessidade de adaptação a uma nova forma de realizar as atividades nesse novo contexto.

Com a finalidade de responder aos objetivos do estudo, inicialmente, foi realizado levantamento dos dados referentes às informações de contato das crianças e adolescentes atendidos no ambulatório de pediatria por meio de acesso ao prontuário.

Para a coleta de dados, foi desenvolvido um questionário com a ferramenta Google Forms ${ }^{\circledR}$ para ser respondido pelas cuidadoras principais. O questionário foi composto por 31 questões, contendo dados sociodemográficos e aspectos relacionados aos cuidados da criança ou adolescente e do cotidiano durante a pandemia. Em seguida, as pesquisadoras entraram em contato com estas pessoas, realizando o convite e envio do formulário. Todos os dados foram computados em uma planilha e após o término da coleta, foi realizada análise qualitativa das questões abertas, bem como a descrição quantitativa dos resultados.

Os participantes da pesquisa tiveram acesso ao Termo de Consentimento Livre e Esclarecido (TCLE) e aceitaram participar do estudo. O projeto de pesquisa foi submetido ao Comitê de Ética em Pesquisa do hospital e aprovado sob o CAAE 33446020.5.0000.5071.

O número total de pacientes atendidos pela equipe multiprofissional na ocasião era $64 \mathrm{e}$ todos foram contatados, com exceção de uma criança que se encontrava em uma instituição de acolhimento. As pesquisadoras não obtiveram sucesso ao contatar 13 cuidadores. Assim, entre as 50 pessoas contatadas, 3 pessoas enfrentaram dificuldades com a internet, impossibilitando Rev. Psicol Saúde e Debate. Out., 2021:7(2): 295-313. 
sua participação, 1 cuidadora recusou a participação e 12 pessoas não completaram o questionário enviado, apesar de terem expressado o desejo em participar. Assim, obteve-se um total de 34 questionários respondidos pelas cuidadoras ${ }^{1}$ principais de crianças e adolescentes com demandas específicas de saúde.

Das crianças e adolescentes acompanhados no ambulatório cujas cuidadoras participaram do estudo, $50 \%(n=17)$ são atendidos entre 1 e 4 anos no ambulatório de pediatria, $38,28 \%(n=13)$ acompanhados há mais de 4 anos e $11,76 \%(n=4)$ atendidos há menos de um ano. É importante destacar que no período de pandemia da COVID-19, 26 (76,47\%) crianças/adolescentes tiveram todas as suas consultas desmarcadas, 4 (11.76\%) tiveram todas as consultas previstas e $3(8,82 \%)$ tiveram parte das consultas canceladas, segundo relato das cuidadoras.

\section{RESULTADOS}

Nesta seção, serão apresentados os resultados do estudo no que se refere à caracterização das pessoas participantes, situação e contexto de vida durante a pandemia, repercussões da pandemia em atividades significativas e estratégias para o enfrentamento dos desafios relacionados ao distanciamento social.

Com relação à caracterização geral das participantes, a Tabela 1, apresenta os dados relacionados às características sociodemográficas das cuidadoras que participaram do estudo.

\begin{tabular}{lcc}
\hline \multicolumn{1}{c}{$\begin{array}{c}\text { Tabela 1- Caracterização das cuidadoras } \\
\text { Variável }\end{array}$} & $\mathbf{n}$ & $\%$ \\
Estado & $\mathbf{n}=\mathbf{3 4}$ & $\%$ \\
Espírito Santo & 34 & 100,0 \\
Idade & $\mathbf{n}=\mathbf{3 3}$ & $\%$ \\
Entre 20-29 anos & 3 & 9,09 \\
Entre 30-39 anos & 17 & 51,52 \\
Entre 40-49 anos & 11 & 33,33 \\
Entre 50-59 anos & 2 & 6,06 \\
Estado Civil & $\mathbf{n}=\mathbf{3 4}$ & $\%$ \\
Casada & 19 & 55,88 \\
Solteira & 13 & 38,24 \\
Divorciada & 2 & 5,88 \\
Escolaridade & $\mathbf{n = 3 3}$ & $\%$ \\
\hline
\end{tabular}

Rev. Psicol Saúde e Debate. Out., 2021:7(2): 295-313. 


\begin{tabular}{lcc}
\hline Ensino Fundamental Incompleto & 7 & 21,21 \\
Ensino Fundamental Completo & 2 & 6,06 \\
Ensino Médio Incompleto & 6 & 18,18 \\
Ensino Médio Completo & 13 & 39,39 \\
Ensino Superior Incompleto & 2 & 6,06 \\
Ensino Fundamental Completo & 2 & 6,06 \\
Pós-graduação & 1 & 3,03 \\
Profissão & $\mathbf{n}=\mathbf{3 3}$ & $\%$ \\
Trabalhadoras informais & 7 & 21,21 \\
Trabalhadoras formais & 12 & 36,36 \\
Do lar/Dona de casa & 13 & 39,39 \\
Estudante & 1 & 3,03 \\
Renda Mensal Familiar & $\mathbf{n}=\mathbf{3 4}$ & $\%$ \\
Não possui & 10 & 29,45 \\
Até R $\$ 1.045,00$ & 19 & 55,88 \\
Entre R $\$ 1.046,00$ e R $\$ 2.090,00$ & 4 & 11,76 \\
Entre R $\$ 2.091,00$ e R $\$ 4.180,00$ & 1 & 2,94 \\
Parentesco & $\mathbf{n}=\mathbf{3 4}$ & $\%$ \\
Mãe & 33 & 97,06 \\
Avó & 1 & 2,94 \\
\hline
\end{tabular}

Fonte: elaborada pelas autoras

Os resultados encontrados em relação aos dados sociodemográficos expostos na Tabela 1 apontam que todas as participantes são do sexo feminino e se identificam como cuidadoras principais das crianças e adolescentes, sendo que metade delas declararam que recebem ajuda para esses cuidados. A maioria das participantes não exerce trabalho remunerado atualmente $(76,47 \%, n=26)$. Quanto à renda mensal, 50,88\% $(n=19)$ das cuidadoras declarou renda de até um salário mínimo, 29,45\% $(n=10)$ declarou não possuir renda, $11,76 \%(n=4)$ declarou renda entre um e dois salários mínimos e 2,94\% $(n=1)$ declarou renda entre dois e quatro salários mínimos.

Em relação à percepção sobre as repercussões sobre o trabalho, a renda e a circulação das famílias durante a pandemia, a Tabela 2 apresenta os resultados obtidos pela pesquisa.

Tabela 2- Situação e contexto de vida com o surgimento da pandemia

Variável

$\mathbf{n}$ $\%$

Rev. Psicol Saúde e Debate. Out., 2021:7(2): 295-313. 


\begin{tabular}{|c|c|c|}
\hline Situação do trabalho durante a pandemia & $\mathrm{n}=34$ & $\%$ \\
\hline $\begin{array}{l}\text { Não trabalhava antes da pandemia, e continuo } \\
\text { sem trabalhar }\end{array}$ & 18 & 52,94 \\
\hline Tive que parar de trabalhar e estou sem renda & 5 & 14,71 \\
\hline Continuei trabalhando normalmente & 5 & 14,71 \\
\hline $\begin{array}{l}\text { Tive que parar de trabalhar, mas continuo } \\
\text { recebendo meu salário }\end{array}$ & 3 & 8,82 \\
\hline Trabalho quando consigo algum serviço & 1 & 2,94 \\
\hline Fui demitida & 1 & 2,94 \\
\hline Recebo auxílio & 1 & 2,94 \\
\hline Meio de realização do trabalho & $\mathrm{n}=\mathbf{3 4}$ & $\%$ \\
\hline Não trabalho & 17 & 50,00 \\
\hline $\begin{array}{l}\text { Parte do trabalho em casa e parte no local de } \\
\text { trabalho }\end{array}$ & 4 & 11,76 \\
\hline Trabalho só remoto & 4 & 11,76 \\
\hline Continuei trabalhando só fora & 8 & 23,53 \\
\hline Trabalha fora só as vezes (faxina) & 1 & 2,94 \\
\hline Impacto da pandemia na renda mensal & $n=34$ & $\%$ \\
\hline Renda foi afetada & 23 & 67,64 \\
\hline Renda não foi afetada & 11 & 32,35 \\
\hline Frequência que sai de casa & $\mathrm{n}=34$ & $\%$ \\
\hline Raramente & 24 & 70,59 \\
\hline Ocasionalmente & 7 & 20,59 \\
\hline Muita frequência & 2 & 5,88 \\
\hline Nunca & 1 & 2,94 \\
\hline Motivos que levam a sair de casa & $\mathrm{n}=39^{*}$ & $\%$ \\
\hline Relacionados com o papel de cuidadora & 29 & 74,36 \\
\hline Trabalho & 8 & 20,51 \\
\hline Visitar familiares & 2 & 5,13 \\
\hline
\end{tabular}

Fonte: elaborada pelas autoras

* $\mathrm{N}$ maior do que a amostra pois cada participante poderia apontar mais de um motivo

Observa-se que o surgimento da pandemia configurou alterações em relação à forma e situação de trabalho das participantes. Ademais, nota-se impacto da pandemia na renda mensal da família, sendo relatado por $67,64 \%(n=23)$ das cuidadoras. As atividades relacionadas ao papel de cuidadora foi um dos principais motivos que levou as cuidadoras a saírem de suas Rev. Psicol Saúde e Debate. Out., 2021:7(2): 295-313. 
casas $(74,36 \%)$, com a frequência variável de "raramente" $(70,59 \%, n=24)$, "ocasionalmente" $(20,59 \%, n=7)$, "muita frequência" $(5,88, n=2)$ e "nunca" $(2,94, n=1)$.

Em relação à rotina de cuidados de saúde com a criança ou adolescente, as participantes relataram alterações causadas pela pandemia relacionadas a: (I) Cancelamento de consultas e mudanças nos protocolos (77,78\%, n=21); (II) Medo de ir em serviços de saúde, onde o risco de contaminação é maior (11,11\%, n=3); e (III) Dificuldades para chegar às consultas $(11,11 \%, n=3)$.

Quanto aos efeitos causados pelo distanciamento social, é possível observar que estresse, ansiedade, tristeza e medo apareceram em maior quantidade, sendo a taxa de prevalência de $82 \%(n=28), 79 \%(n=27), 55 \%(n=19)$ e $52 \%(n=18)$ respectivamente. Esses sentimentos foram seguidos por desespero $(41 \%, n=14)$, raiva $(26 \%, n=9)$, confiança $(23 \%, n=8)$, frustração $(20 \%, n=7)$ e esperança $(20 \%, n=7)$. Já empatia e desesperança aparecem em menor quantidade, sendo a taxa de prevalência 14\% $(n=5)$ e 11\% $(n=4)$ na ordem citada.

O impacto provocado pela pandemia nas atividades é apresentado na Tabela 3 , demonstrando as repercussões nas atividades significativas e as estratégias desenvolvidas pelas cuidadoras para lidar com o impacto negativo do distanciamento social.

Tabela 3- Atividades e pandemia

Variável $\mathbf{n} \quad \%$

Repercussões da pandemia em atividades significativas

\section{Atividades significativas}

Atividades de lazer

Visitar familiares e amigos

Atividades indispensáveis

Atividades de expressão religiosa

Atividade física

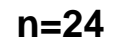

7

6

6

4

1
$\%$

29,17

25,00

25,00

16,67

4,17

\section{Estratégias utilizadas pelas participantes}

\section{Estratégias que deram certo}

$\mathrm{n}=\mathbf{2 3}$

$\%$

Atividades de lazer 12

52,17

Atividades gerais de prevenção

Uso de recursos visuais

Reestruturação da rotina

Espiritualidade

Estudos

Ajuda profissional

1

4,35

Estratégias que não deram certo

1

$n=15$

$\%$

Rev. Psicol Saúde e Debate. Out., 2021:7(2): 295-313. 
Estratégias gerais de prevenção

Habilidades pessoais de enfrentamento

Atividades de lazer no domicílio

Atividades relacionadas ao cuidado com a casa

Acompanhamento das informações da

pandemia

Atividades escolares no domicílio

Atividades em família

Não souberam responder o que não deu certo

3

20,00

3

20,00

2

13,33

2

2

1

6,67

1

6,67

1

6,67

Fonte: elaborada pelas autoras

Em relação aos desafios trazidos pela pandemia, as respostas das participantes foram agrupadas em 6 categorias: (I) Relação negativa com o vírus (22,5\%, $n=9)$; (II) Distanciamento Social (25,0\%, $n=10)$; (III) Impacto Financeiro (10,0\%, $n=4)$; (IV) Ruptura com atividades cotidianas (25,0\%, $n=10)$; (V) Medidas de prevenção (10,0\%, $n=4)$; e (VI) Sobrecarga do cuidador $(7,5 \%, n=3)$.

Quanto ao desenvolvimento de estratégias de enfrentamento para amenizar os efeitos do distanciamento social, $61,76 \%(n=21)$ das participantes afirmaram ter encontrado estratégias. Segundo os dados coletados, $38,10 \%(n=8)$ das participantes conseguiram desenvolver suas próprias estratégias, $38,10 \% \quad(n=8)$ encontraram dicas em redes sociais, $9,52 \% \quad(n=2)$ encontraram dicas em sites da internet, 9,52\% $(n=2)$ obtiveram dicas com amigos e familiares e $4,76 \%(n=1)$ contaram com ajuda profissional.

Parte das cuidadoras participantes $(26,47 \%, n=9)$ informou que começaram a fazer alguma atividade nova ou retomaram uma atividade durante a pandemia, sendo $88,89 \%(n=8)$ relacionadas ao lazer, bem-estar e relacionamento familiar e $11,11 \%(n=1)$ relacionadas ao papel do cuidador.

A respeito da opinião dos familiares participantes sobre as consequências da pandemia da COVID-19 na saúde da criança/adolescente $64,7 \%(n=22)$ dos participantes afirmam que a saúde foi prejudicada, enquanto $35,29 \%(n=12)$ referem que não houve prejuízos.

\section{DISCUSSÃO}

As mudanças sociais relacionadas às mulheres são frutos de um longo e complexo processo de transformações no âmbito micro e macrossocial (Cavenaghi \& Alves, 2018). Porém, ainda existem na sociedade valores que reforçam estereótipos de gênero que resultam em impactos nos papéis ocupacionais da mulher e no seu cotidiano. Majoritariamente os cuidados Rev. Psicol Saúde e Debate. Out., 2021:7(2): 295-313. 
domésticos e familiares de dependentes continuam sendo responsabilidades das mulheres e as divisões mais igualitárias de papéis ainda é uma realidade em construção (Verza, Sattler, \& Strey, 2015).

Tal afirmação pode ser observada nos dados apresentados acima, onde é possível verificar que todas as participantes da pesquisa são do sexo feminino, identificando-se como cuidadora principal de crianças e adolescentes com necessidades específicas de saúde. Notase a presença da configuração familiar em que a mulher se divide entre diversos papéis ocupacionais, ficando responsável pelos cuidados com seus filhos e/ou netos e da casa, além de gerir seu autocuidado, e realizar atividades remuneradas que garantam o sustento e a manutenção do lar, corroborando com outros estudos que abordam essa temática (Loyola, 2020; Moreira, Alves, Oliveira, \& Natividade, 2020).

Historicamente, no processo de saúde/doença, associa-se o cuidado à figura feminina, mais especificamente à figura materna. Sendo assim, as mulheres assumem diversos papéis determinados e impostos pelo meio sócio-histórico-cultural (Wegner \& Pedro, 2010). O cuidado de crianças e adolescentes com condições específicas de saúde acrescenta atividades complexas e específicas ao cotidiano das cuidadoras, contribuindo para o aumento da sobrecarga que interfere na qualidade de vida das cuidadoras em diversas áreas (Macedo, Paiva, \& Ramos, 2015). Comumente chamadas de mães "guerreiras" ou "especiais", a condição em que essas mulheres desempenham suas ocupações relacionadas ao papel da maternidade se relacionam aos esforços para garantir o cuidado de seus filhos mesmo inseridas em um contexto que reforçam sua vulnerabilidade (Dias, Berger, \& Lovisi, 2020).

Cabe ressaltar que o processo de ser cuidadora envolve aspectos objetivos como alterações na rotina e na vida social, perdas financeiras e envolvimento em excessivo número de tarefas, além dos aspectos subjetivos relacionados com percepções, expectativas e pensamentos positivos e negativos do cuidador (Barros et al., 2017).

De acordo com o Censo Demográfico do Instituto Brasileiro de Geografia e Estatísticas (IBGE, 2010), o Brasil possui 37,3\% famílias chefiadas por mulheres, ou seja, a obtenção da renda familiar e cuidados são responsabilidades da figura feminina da família. Nesta pesquisa, 19 mulheres estão inseridas no mercado de trabalho, sendo $12 \mathrm{em}$ trabalho formal e 7 em trabalho informal. Dentre as 33 participantes, 13 se definem como donas de casa e 1 como estudante.

Com avanço neoliberal houve um aumento dos empregos informais, terceirizações e trabalhos em tempo parcial e estudos demonstram que as mulheres estão mais sujeitas às relações de precariedade do trabalho quando comparado com a população masculina, fator que causa impactos negativos na saúde das mulheres (Brito, 2000; Tavares, 2019). As mulheres Rev. Psicol Saúde e Debate. Out., 2021:7(2): 295-313. 
detêm maior índice de desemprego, diferença salarial e estão mais inseridas no mercado informal da economia, e consequentemente vivenciam uma carga horária maior que a do homem (Brito, 2000). Esses dados refletem a divisão sexual do trabalho, que é resultado das relações de gênero historicamente construídas e adaptadas para as sociedades, e se caracteriza pelo envolvimento dos homens em atividades da esfera produtiva e das mulheres em atividades da esfera reprodutiva (Dias et al., 2020).

Em relação a situação de trabalho das participantes da pesquisa com a pandemia, identifica-se que apenas 5 participantes mantiveram seus empregos sem alterações, 3 participantes continuaram recebendo seus salários, porém não estavam exercendo as tarefas relacionadas ao emprego no momento da pesquisa, 5 mulheres relataram que ficaram impossibilitadas de conseguirem novos trabalhos durante a pandemia e 1 participante informou que foi demitida de seu emprego. Por fim, 67,64\% ( $n=23)$ das participantes consideraram que a pandemia afetou a renda da família.

Mesmo com a necessidade de adotar o distanciamento social, grande parte dos trabalhadores, formais e informais, não puderam adotar o isolamento domiciliar como meio de prevenção à COVID-19, pela necessidade de cumprir a jornada de trabalho presencial para manutenção da renda, além de casos de desemprego e diminuição abrupta da renda familiar. Dessa forma, observa-se uma ruptura na rotina e no contexto socioeconômico que foi vivenciada de forma heterogênea pela população brasileira, gerando diferentes implicações na vida dos indivíduos (Fiocruz, 2020; Trovão, 2020; Macedo, 2020).

Tal afirmação é possível ser observada através dos resultados, onde metade das participantes que mantiveram seus empregos continuaram exercendo suas atividades do trabalho presencialmente $(n=8)$. A outra metade se divide em participantes que mantiveram todas as suas atividades relacionadas ao trabalho de forma remota $(n=4)$ e em participantes que se enquadram na categoria de trabalho mista (remoto e presencial) $(n=4)$.

No mundo do trabalho, as mulheres dedicam-se tanto quanto os homens, porém, não é a única jornada de trabalho que têm sob suas responsabilidades. Ao retornarem ao domicílio, enfrentam de forma intensa o trabalho doméstico e de cuidados com os familiares (Probst \& Ramos, 2003). As representações de gênero podem contribuir para as desigualdades, uma vez que a mulher está associada à esfera privada, ou seja, as tarefas do domicílio (privado e invisível) não são valorizadas, pois não resultam em valor produtivo ao mercado de trabalho (Zimmermann, Vicente, \& Machado, 2021). Logo, as múltiplas jornadas de trabalho enfrentadas pelas mulheres resultam na sobrecarga e prejuízos para a saúde física e mental.

Constata-se que é urgente o aprofundamento das discussões sobre a relação das mulheres e trabalho. $O$ trabalho não-remunerado de cuidar, não é colocado em foco, uma vez Rev. Psicol Saúde e Debate. Out., 2021:7(2): 295-313. 
que o cuidado é indispensável e inerente à existência da humanidade. A valorização da economia do cuidado é fundamental para proporcionar que as mulheres consigam conciliar suas carreiras e famílias, sem sobrecarga e consequências para sua saúde (Zimmermann, Vicente, \& Machado, 2021) e seus outros possíveis projetos de vida. Durante a pandemia, verificou-se o fechamento de instituições que auxiliavam as cuidadoras no manejo do cuidado, reverberando no desempenho de suas atividades remuneradas e aumento da sobrecarga no domicílio. Ademais, não foram traçadas estratégias que visam o apoio das famílias, em especial das crianças com necessidades específicas de saúde, no enfrentamento das consequências causadas pela pandemia.

Outro desafio decorrente da pandemia identificado na pesquisa foi o distanciamento social. As atividades relacionadas à interação social com outras pessoas (familiares, amigos, comunidade e outros) compõem a ocupação "participação social" e é estruturante do cotidiano dos indivíduos (AOTA, 2020). De acordo com os dados coletados, a pandemia provocou a diminuição e/ou interrupção da circulação nos espaços da comunidade e consequentemente a ruptura do convívio social, impactando negativamente a participação social. A maioria das pessoas se voltou para o núcleo familiar, restringindo-se ao ambiente domiciliar, o que resultou em alterações no cotidiano.

Estudos recentes demonstraram que as medidas de prevenção contra o novo coronavírus, em especial o distanciamento e isolamento social, têm mostrado efeitos psicológicos negativos, como sintomas de Transtorno do Estresse Pós-Traumático (TEPT), depressão, estresse, ansiedade e medo (Raony, Figueiredo, Pandolfo, Araujo, Bomfim, \& Savino, 2020). Cabe ressaltar que esse impacto pode ser influenciado pela história do sofrimento psíquico anteriormente identificado, trabalho na área da saúde, tempo de quarentena prolongado, medo da infecção, suprimentos e informações inadequadas e recursos financeiros escassos (Raony et al., 2020). Para além da importância da interação social na vida humana, no contexto de famílias com crianças e adolescentes com demandas específicas de saúde, o distanciamento apresenta impactos negativos, pois há uma dificuldade na manutenção da rede de apoio, essencial para a diminuição da sobrecarga do cuidador principal.

Ao analisar os resultados da pesquisa ao que se refere aos efeitos psicossociais do distanciamento social e os desafios trazidos pela pandemia, as participantes relataram dificuldades com a adoção do distanciamento social, impacto financeiro, ruptura com atividades cotidianas, cumprimento das medidas de prevenção e sobrecarga do cuidador. Além disso, foi identificada maior prevalência de ansiedade, tristeza e medo, seguidos por desespero, raiva, confiança e frustração. 
Rocha et al. (2021) identificou que as repercussões psicossociais resultantes das medidas de distanciamento social foram frequentes em diversos países, indicando impactos negativos na saúde mental e na qualidade de vida. Além disso, os comprometimentos psicológicos foram associados a instabilidades de humor, níveis elevados de ansiedade, situação de estresse, frustração, solidão, raiva e alteração de sono. Por fim, segundo o estudo, o grau de sofrimento psíquico também foi relacionado com duração prolongada das medidas de distanciamento social, instabilidades econômicas e incertezas sobre a doença, contexto que se agudiza no Brasil.

De acordo com os resultados expostos, a maior parte $(61,76 \%, n=21)$ das participantes encontrou estratégias para amenizar os efeitos do distanciamento social, sendo avaliadas positivamente pelas participantes o envolvimento em atividades de lazer, seguido pela adoção das medidas de prevenção, uso de recursos virtuais, reestruturação da rotina, espiritualidade, estudos e ajuda profissional.

Conforme relatado nos resultados, o lazer foi considerado como uma das categorias mais prejudicadas com a pandemia. O lazer é uma ocupação que pode ser caracterizada como uma atividade de caráter não obrigatório e intrinsecamente motivada, na qual o indivíduo se engaja de forma livre, sem relação com outras ocupações obrigatórias, como o trabalho (AOTA, 2020).

O lazer é um direito social garantido pela constituição de 1988, entretanto, existem parcelas da população que encontram dificuldades de acesso a esse direito. Pesquisas realizadas no Brasil identificam alguns fatores que contribuem para a apropriação desigual do lazer como faixa etária, economia e gênero. Em relação ao último fator, nota-se que as mulheres possuem menos acesso ao lazer devido a sua rotina do trabalho doméstico, obrigações familiares e/ou jornada dupla de trabalho (Silva, Stoppa, Isayama, Marcellino, \& Melo, 2011).

Com base no exposto, é possível observar um comprometimento significativo em relação a qualidade de vida das cuidadoras, uma vez que o envolvimento em atividades de lazer contribui para a diminuição da sobrecarga, e está intimamente relacionado à saúde.

O impacto da pandemia no lazer também foi evidenciado ao abordar as repercussões nas atividades significativas, em que as participantes relataram alterações nas atividades de lazer, atividades de expressão religiosa, visitar familiares e amigos, atividades indispensáveis e atividade física. Parte das cuidadoras (26,47\%) começaram a fazer alguma atividade nova ou retomaram uma atividade durante a pandemia, relacionadas ao lazer, bem-estar, relacionamento familiar e relacionadas ao papel do cuidador. Tais resultados demonstram o impacto da pandemia nas ocupações humanas e as estratégias que os indivíduos encontraram para amenizar os efeitos do distanciamento social. 
Outro dado relevante obtido através da pesquisa, está relacionado ao tratamento de saúde das crianças e dos adolescentes. A maioria das cuidadoras acredita que a pandemia prejudicou o acompanhamento de saúde. Destaca-se que 76,47\% ( $n=26)$ das crianças e adolescentes tiveram todas suas consultas canceladas, $8,82 \%(n=3)$ tiveram parte de suas consultas mantidas e apenas $11,76 \%(n=4)$ foram atendidas normalmente. Diversas são as ocupações que permeiam o papel ocupacional do cuidador, sendo o tratamento de saúde uma delas. As crianças e adolescentes com necessidades específicas de saúde possuem como característica um processo de tratamento contínuo e que, em muitos casos, trazem mudanças no estilo de vida tanto na criança e adolescente, quanto em seu núcleo familiar (Kudo, Barros, \& Joaquim, 2017).

Muitos serviços tiveram seus atendimentos suspensos inicialmente, devido ao desconhecimento quanto ao comportamento do vírus e necessidade de distanciamento social para diminuição da propagação da doença. Posteriormente, fez-se necessária a adoção de protocolos para continuidade dos serviços, porém, muitos usuários foram prejudicados tanto com a interrupção, quanto com o retorno restrito, considerando, neste caso a necessidade de acompanhamento sistemático e constante das pessoas com necessidades específicas de saúde. Situação observada nesta pesquisa, na qual a ausência do acompanhamento pelos serviços de saúde e possíveis consequências no quadro clínico e desenvolvimento das crianças e dos adolescentes é uma preocupação entre as cuidadoras.

Frente a esse cenário, verifica-se a necessidade de planejamento, políticas públicas para a garantia de condições estruturais, planejamento e práticas gerenciais que permitam a continuação dos tratamentos de saúde, garantindo a proteção dos usuários, familiares e profissionais, mas também a diminuição das consequências que podem surgir com a interrupção dos acompanhamentos de saúde (Imperatori \& Bezerra, 2020; Santos, et al., 2020).

\section{CONSIDERAÇÕES FINAIS}

Este estudo objetivou investigar os efeitos da pandemia da COVID-19 na vida de cuidadoras de crianças e adolescentes com necessidades específicas de saúde, identificando o impacto do distanciamento social em relação ao perfil ocupacional de cuidadoras de crianças e adolescentes acompanhados por uma equipe multiprofissional.

O estudo evidenciou a sobrecarga das mulheres no que se refere ao desempenho de múltiplos papéis ocupacionais, acentuada pelas medidas de distanciamento social. Além disso, foi identificada a falta de rede de apoio e recursos financeiros para enfrentamento do período da pandemia. Em relação às ocupações, os resultados desse estudo apontam para a intensificação Rev. Psicol Saúde e Debate. Out., 2021:7(2): 295-313. 
do papel de cuidadora, ausência da participação em atividades de lazer e a importância do envolvimento em atividades significativas que apoiam e sustentam a saúde. Por fim, foi identificado que a pandemia também prejudicou o acesso aos serviços de saúde.

Aponta-se a necessidade do desenvolvimento de estratégias que sustentam as redes de apoios das famílias, mesmo aquelas aplicadas à distância, uma vez que momentos de crise geram situações que requerem aumento dos recursos e auxílios para enfrentamento. Ressaltase também a necessidade dos serviços de saúde e educação desenvolverem estratégias efetivas para alcançar a população do seu território e garantir o direito de acesso a estes serviços em situações de grave crise social.

\section{REFERÊNCIAS}

American Occupational Therapy Association. (2020). Occupational therapy practice framework: domain and process $\left(4^{\circ}\right.$ ed.). American Journal of OccupationalTherapy, 74(2), 1-87. https://doi.org/10.5014/ajot.2020.74S2001

Araújo, M. G. O., Dutra, M. O. M., Freitas, C. C. S. L., Guedes, T. G., Souza, F. S., Baptista, R. S. (2019). Cuidando de quem cuida: qualidade de vida e sobrecarga de mulheres cuidadoras. Revista Brasileira de Enfermagem, 72(3), 728-736. https://doi.org/10.1590/0034-7167-2018-0334

Ballarin, M. L. G. S., Benedito, A. C., Krön, C. A., Christovam, D. (2016). Perfil sociodemográfico e sobrecarga de cuidadores informais de pacientes assistidos em ambulatório de terapia ocupacional. Cadernos Brasileiros de Terapia Ocupacional, 24(2), 315-321. https://doi.org/10.4322/0104-4931.ctoAO0607

Barros, A. L. O., Barros, A. O., Barros, G. L. M., Santos, M. T. B. R. (2017). Sobrecarga dos cuidadores de crianças e adolescentes com Síndrome de Down. Ciência \& Saúde Coletiva, 22, 3625-3634. https://doi.org/10.1590/1413$\underline{812320172211.31102016}$

Barros, D. D., Lopes, R. E., Galheigo, S. M. (2002). Projeto Metuia- Terapia Ocupacional no campo social. O Mundo da Saúde, 26(3), p. 365-369. https://pesquisa.bvsalud.org/portal/resource/pt/lil-366553

Brito, J. C. (2000). Enfoque de gênero e relação saúde/trabalho no contexto de reestruturação produtiva e precarização do trabalho. Cadernos de Saúde Pública, 16(1), 195-204. https://doi.org/10.1590/S0102-311X2000000100020

Cavenaghi, S. (2018). Mulheres chefes de família no Brasil: avanços e desafios (1ํed). Rio de Janeiro: ENS-CPE. Recuperado de https://www.ens.edu.br/arquivos/mulheres-chefes-de-familia-no-brasil-estudosobre-seguro-edicao-32 1.pdf 
Costa, E. F., Oliveira, L. S. M., Corrêa, V. A. C., Costa, O. A. A. C. (2017). Ciência ocupacional e terapia ocupacional: algumas reflexões. Revista Interinstitucional Brasileira de Terapia Ocupacional, 1(5), 650-663. Recuperado de https://revistas.ufrj.br/index.php/ribto/article/view/9687/pdf

Dias, F. M., Berger, S. M. D., Lovisi, G. M. Mulheres guerreiras e mães especiais? Reflexões sobre gênero, cuidado e maternidades no contexto de pós-epidemia de zika no Brasil. Physis: Revista de Saúde Coletiva, Rio de Janeiro, v. 30(4), e300408, 2020. https://doi.org/10.1590/S0103-73312020300408

Fiocruz, Saúde Mental e Atenção Psicossocial na Pandemia COVID-19. Crianças na Pandemia COVID-19. (2020). Recuperado de https://www.fiocruzbrasilia.fiocruz.br/wpcontent/uploads/2020/05/crianc\%cc\%a7as pandemia.pdf

Imperatori, T. K., Bezerra, F. P. 2020. Notas introdutórias sobre o direito à vida das pessoas com deficiência no contexto de COVID-19. Revista Observatório, 6(3), 1-15. https://doi.org/10.20873/uft.2447-4266.2020v6n3a8pt

Instituto Brasileiro de Geografia e Estatísticas. 2010. Estatísticas de Gênero.

Lei $\mathrm{n}^{\circ} 13.146$ de 6 de julho de 2015. Institui a Lei Brasileira de Inclusão da Pessoa com Deficiência (Estatuto da Pessoa com Deficiência). Recuperado de http://www.planalto.gov.br/ccivil 03/ ato2015-2018/2015/lei/l13146.htm

Loyola, M. A. (2020). Covid-19: uma agenda de pesquisa em torno das questões de gênero. Physis: Revista de Saúde Coletiva, 30(3), e300312. Epub September 04, 2020. https://doi.org/10.1590/s0103-73312020300312

Kristensen, H. K., Petersen, K. S. (2016). Occupational science: An important contributor to occupational therapists' clinical reasoning. Scandinavian Journal of Occupational Therapy, 23(3)240-243. https://doi.org/10.3109/11038128.2015.1083054

Kudo, A. M., Barros, P. B. M., Joaquim, R. H. V. T. (2017). Terapia Ocupacional em Enfermaria Pediátrica. In Carlo, M. M. R. P., Kudo, A. M. Terapia Ocupacional em contextos hospitalares e cuidados paliativos. (p. 127-145). Editora Payá

Macedo, E. C., Silva, L. R., Paiva, M. S., Ramos, M. N. P. (2015). Sobrecarga e qualidade de vida de mães de crianças e adolescentes com doença crônica: revisão integrativa. Revista Latino-Americana de Enfermagem, 23(4), 769-777. https://doi.org/10.1590/0104-1169.0196.2613

Macedo, E. E. (2020). Desigualdade e pandemia nas vidas dos brasileirinhas e brasileirinhos. Zero-a-seis, 22(especial), 1404-1419. https://periodicos.ufsc.br/index.php/zeroseis/article/view/77746

Maior, I. M. M. L. (2017). Movimento político das pessoas com deficiência: reflexões sobre a conquista de direitos. Inclusão Social, 10(2), 28-36. Recuperado de http://revista.ibict.br/inclusao/article/view/4029

Maciel, E. L. N., Melo, D. C. F., Delcarro, J. C. S., Robaina, I. M. M., Jabor, P. M., Junior, E. G., Zandonade, E. (2020). Pessoas Com Deficiência e COVID-19 no

Rev. Psicol Saúde e Debate. Out., 2021:7(2): 295-313. 
estado do Espírito Santo: Entre a invisibilidade e a falta de Políticas Públicas. SciELO Preprints. https://doi.org/10.1590/SciELOPreprints.1540

Moreira, L. E., Alves, J. S., Oliveira, R. G., Natividade, C. (2020). Mulheres em tempos de pandemia: um ensaio teórico-político sobre a casa e a guerra. Psicologia \& Sociedade, 32, e020014. Epub September 04, 2020. https://doi.org/10.1590/1807-0310/2020v32240246

Oliveira, A. (2020). A espacialidade aberta e relacional do lar: a arte de conciliar maternidade, trabalho doméstico e remoto na pandemia da COVID-19. Revista Tamoios, 16(1). doi: https://doi.org/10.12957/tamoios.2020.50448

Organização Panamericana de Saúde. (2020). Transmissão do SARS-CoV-2: implicações para as precauções de prevenção de infecção. Resumo científico, Recuperado de https://iris.paho.org/bitstream/handle/10665.2/52472/OPASWBRACOVID1920089 por.pdf? sequence $=1$ \&isAllowed $=y$

Poellnitz, J. C. V. (2018). Atividade, cotidiano e ocupação na terapia ocupacional no Brasil: usos e conceitos em disputa. Dissertação de mestrado Programa de PósGraduação em Terapia Ocupacional da Universidade Federal de São Carlos. São Carlos, Brasil.

Raony, Í., Figueiredo, C. S., Pandolfo, P., Araujo, E. G., Bomfim, P. O. S., Savino, W. (2020). Psycho-neuroendocrine-immune interactions in COVID-19: potential impacts on mental health. Frontiers in Immunology, 11, 1170. https://doi.org/10.3389/fimmu.2020.01170

Rocha, D. M. S., Silva, J. S., Abreu, I. M., Mendes, P. M., Leite, H. D. C. S., Ferreira, M. C. S. (2021). Efeitos psicossociais do distanciamento social durante as infecções por coronavírus: revisão integrativa. Acta Paulista de Enfermagem, 34, eAPE01141. Epub March 15, 2021.https://doi.org/10.37689/actaape/2021ar01141

Salles, M. M., Matsukura, T. S. (2013). Estudo de revisão sistemática sobre o uso do conceito de cotidiano no campo da terapia ocupacional no Brasil. Cadernos de Terapia Ocupacional, 21(2), p. 265-273. http://dx.doi.org/10.4322/cto.2013.028

Salles, M. M., Matsukura, T. S. (2016). O uso dos conceitos de ocupação e atividade na Terapia Ocupacional: uma revisão sistemática da literatura. Cadernos de Terapia Ocupacional, 24(4), 801-810. http://dx.doi.org/10.4322/01044931.ctoAR0525

Santos, J. L. G., Lanzoni, G. M. M., Costa, M. F. B. N. A., Debetio, J. O., Sousa, L. P., Santos, L. S., Marcelino, T. B., Mello, A. L. S. F. (2020). Como os hospitais universitários estão enfrentando a pandemia de COVID-19 no Brasil?. Acta Paulista de Enfermagem, 33. https://doi.org/10.37689/acta-ape/2020ao01755

Silva, D. A. M., Stoppa, E. A., Isayama, H. F., Marcellino, M. C., Melo, V. A. A. (2011). Importância da recreação e do lazer. In I R. P. Rodrigues C. R. Bonalume (Orgs.), Cadernos interativos - elementos para o desenvolvimento de políticas, programas e projetos intersetoriais, enfatizando a relação lazer, escola e

Rev. Psicol Saúde e Debate. Out., 2021:7(2): 295-313. 
processo educativo (4; 52). Gráfica e Editora Ideal. Recuperado de https://repositorio.ufsc.br/bitstream/handle/123456789/128023/CADERNO\%20IN TERATIVO\%204.pdf?sequence $=1$

Sassaki, R. K. (2009). Inclusão: Acessibilidade no lazer, trabalho e educação. Revista Nacional de Reabilitação (Reação). 10-16. Recuperado de https://files.cercomp.ufg.br/weby/up/211/o/SASSAKI Acessibilidade.pdf?1473203319

Tavares, J. D. (2020). O trabalho da mulher e a sobrecarga emocional. Trabalho de Conclusão de Curso, Universidade Regional do Noroeste do Estado do Rio Grande do Sul. Rio Grande do Sul, Brasil. Recuperado de: https://bibliodigital.unijui.edu.br:8443/xmlui/handle/123456789/6554

Trovão, C. J. B. M. (2020). A Pandemia da Covid-19 e a Desigualdade de Renda no Brasil: Um Olhar Macrorregional para a Proteção Social e os Auxílios Emergenciais. Universidade Federal do Rio Grande do Norte. Recuperado de https://ccsa.ufrn.br/portal/wp-content/uploads/2020/05/TROV\%C3\%83O-2020PANDEMIA-E-DESIGUALDADE.pdf

Verza, F., Sattler, M. K., Strey, M. N. (2015). Mãe, mulher, chefe de família: perspectivas de gênero na terapia familiar. Pensando famílias, 19(1), 46-60. Recuperado de http://pepsic.bvsalud.org/scielo.php?pid=S1679494X2015000100005\&script=sci abstract\&tlng=pt

Wegner, W., Pedro, E. N. R. (2010). Os múltiplos papéis sociais de mulheres cuidadoras-leigas de crianças hospitalizadas. Revista Gaúcha de Enfermagem, 31(2), 335-342. https://doi.org/10.1590/S1983-14472010000200019

Zimmermann, T. R., Vicente, J. A. P., Machado, A. A. (2021). Análise de gênero a partir da economia do cuidado em tempos de pandemia: estudo de caso de mulherescuidadoras de crianças em CEMEI. Brazilian Journal of Development, 7(3), p. 26092-26112. https://doi.org/10.34117/bjdv7n3-353

' Considerando que todos os participantes se identificam pelo gênero feminino, adotamos o termo "cuidadoras" para tratar sobre as participantes do estudo. 